\title{
The role of perceived behavioural control as mediator on financial Incentives towards saving in a voluntary retirement fund
}

\begin{abstract}
The purpose of the mediation analysis is to investigate how perceived behavioural control mediates the relationship between the cash and tax incentives towards the intention to save in a voluntary retirement fund. A quantitative approach was adopted analysing 384 responses collected through a nationwide multistage proportionate cluster sampling. A nonparametric sampling procedure using bootstrapping following the Preacher and Hayes technique in mediating analysis were employed. The specific indirect effect from the bootstrapping result concluded that tax incentives influence the saving intention via perceived behavioural control, in contrast to cash incentives which is not significant. Predicting a person's intention to save in a voluntary retirement fund is an important issue, and the findings of this study would have practical implications on policy makers and commercial marketers alike, as it would help to encourage retirement savings through voluntary funds to prevent financial insufficiency in the golden age.
\end{abstract}

Keyword : Voluntary retirement fund; Social security; Retirement savings; Financial incentives 